\title{
A Fourier Ellipsometer Using Rotating Polarizer and Analyzer at a Speed Ratio $1: 1$
}

\author{
Taher M. El-Agez and Sofyan A. Taya \\ Physics Department, Islamic University of Gaza, P.O. Box 108, Gaza, Palestine \\ Correspondence should be addressed to Sofyan A. Taya, staya@iugaza.edu.ps \\ Received 6 September 2010; Accepted 26 November 2010 \\ Academic Editor: S. C. Mukhopadhyay
}

Copyright (๑) 2010 T. M. El-Agez and S. A. Taya. This is an open access article distributed under the Creative Commons Attribution License, which permits unrestricted use, distribution, and reproduction in any medium, provided the original work is properly cited.

We propose theoretically a spectroscopic ellipsometer in which the polarizer and the analyzer are rotating synchronously in the same direction with the same angular speed. The light intensity received by the detector contains four components, one dc and three AC terms, with frequencies of $2 \omega, 4 \omega$, and $6 \omega$. The main advantage of the proposed ellipsometer is that one can extract the ellipsometric parameters $\psi$ and $\Delta$ from the AC Fourier coefficients without relying on the dc component which is considered to be a serious problem in rotating-analyzer or -polarizer ellipsometers. This allows measurements in a semidark room without worrying about stray light problems, dark currents in detectors, and long-term fluctuations in light sources. The results from the simulated spectra of the complex refractive index of c-Si and Au are presented. The noise effect on the proposed ellipsometer was simulated and plotted for the two samples.

\section{Introduction}

Ellipsometry has proven to be an extremely powerful tool for the study of the optical properties of materials with high optical data precision in measurement. Being accurate, fast and nondestructive in data acquisition and characterization of the samples, ellipsometry has been widely applied especially in the scientific research labs and microelectronic industry with continuous efforts and progress made by developing different theoretical models and experimental techniques [1-5].

An ellipsometric measurement allows one to quantify the phase difference between $\mathbf{E}_{p}$ and $\mathbf{E}_{s}, \Delta$, and the change in the ratio of their amplitudes given by $\tan \psi$. For a reflecting surface, the forms of $\Delta$ and $\psi$ are

$$
\Delta=\delta_{p}-\delta_{s}, \quad \tan \psi=\frac{\left|\mathrm{r}_{p}\right|}{\left|\mathrm{r}_{s}\right|}
$$

where $\delta_{p}$ and $\delta_{s}$, are the phase changes for the $p$ and $s$ components of light and $r_{p}$ and $r_{s}$ are the complex Fresnel reflection coefficients: for the $p$ and $s$ components which may be written as

$$
\begin{aligned}
& r_{p}=\rho_{p} e^{i \delta_{p}}, \\
& r_{s}=\rho_{s} e^{i \delta_{s}} .
\end{aligned}
$$

The expressions for $r_{p}$ and $r_{s}$ for a single interface between medium 0 (ambient), with a complex refractive index $N_{0}$, and medium 1 (substrate), with a complex refractive index $N_{1}$ are given by [1]

$$
\begin{aligned}
& r_{p}=\frac{N_{1} \cos \theta_{0}-N_{0} \cos \theta_{1}}{N_{1} \cos \theta_{0}+N_{0} \cos \theta_{1}}, \\
& r_{s}=\frac{N_{0} \cos \theta_{0}-N_{1} \cos \theta_{1}}{N_{0} \cos \theta_{0}+N_{1} \cos \theta_{1}},
\end{aligned}
$$

where $\theta_{0}$ and $\theta_{1}$ are the angles of incidence and refraction.

Once, $\psi$ and $\Delta$ are measured at a given wavelength one can invert Fresnel equations to extract the optical parameters of a bulk sample. For a substrate/thin film/ambient structure, one must at least measure at several wavelengths and require that the same value of the film thickness $d$ gives the best fit for the real and imaginary parts of the film index $n$ and $k$ at each wavelength, or, yet, measure spectra at at least two different 
angles of incidence. If the film is known to have $k=0$, then $n$ and $d$ can be found from one measurement.

Early in the seventies, the dynamic scanning ellipsometer has shown a great ability of determining optical constants of materials in the range of $1.5-6 \mathrm{eV}$ photon energy $[6,7]$. Since then, ellipsometry has received an increasing interest and the technique has been improved. The simplest and most widely available Fourier ellipsometer uses a single rotating polarizer or analyzer to measure $\psi$ and $\Delta$ of an isotropic reflecting surface. Different models that can work in the visible region have been proposed and constructed [8-12]. Among the various configurations of spectroscopic ellipsometers commonly used is the rotating analyzer ellipsometer RAE [6]. In such a model, the polarizer angle $P$ is fixed while the analyzer angle $A$ rotates at an angular speed $\omega$. In terms of $A=\omega t$, the intensity of light emerging from the analyzer can be written as one dc and two ac components from which the ellipsometric parameters $\psi$ and $\Delta$ are obtained. The RAE has the advantage of simple system design but it involves the dc component which causes a serious problem. The reduction of the dc background requires particular techniques and the calibration of such system is also time consuming [13]. Azzam [14] suggested synchronously rotating both the polarizer and the analyzer with the ratio of $1: 3$. In his work, the intensity of light reaching the detector includes four cosine and four sine terms. His system suffers a lack of clear explanation of each coefficient and its relation to the ellipsometric parameters $\psi$ and $\Delta[10]$.

In 1987, a rotating polarizer and analyzer RPA spectroscopic ellipsometer was proposed and constructed [10]. In the design, the polarizer and the analyzer rotate with a speed ratio $1: 2$. The final light intensity then contains three cosine terms from which $\psi$ and $\Delta$ are calculated. In this design, the errors arising from the phase shift and dc background are eliminated.

An improved RPA spectroscopic ellipsometer was proposed [11,12] with the speed ratio still being $1: 2$ but with the incident angle being fully variable. Moreover, a fixed polarizer was placed in the optical path to eliminate the source polarization effect. The final light intensity thus contains four ac components. The optical constants and the ellipsometric parameters were obtained by calculating any one of the two sets of ac signals.

An et al., have developed RPA ellipsometer in which the polarizer and the analyzer rotate in the same direction with the same angular speed [15] but their study lacks the extraction of the optical parameters from the ellipsometric parameters. In their study, the ellipsometric parameters were obtained using two ac coefficients and the de term.

In this work, we present theoretically a rotating polarizeranalyzer (RPA) ellipsometer in which the linear polarizer and the linear analyzer are rotating synchronously at a ratio of $1: 1$ in the same direction with an isotropic sample placed between these two rotating elements. Consequently, the intensity of the light measured by the detector is modulated by the mechanical rotation of these elements and by the reflection from the sample surface. The Fourier spectrum of this signal includes seven coefficients; the dc component, the first harmonics, the second harmonics,

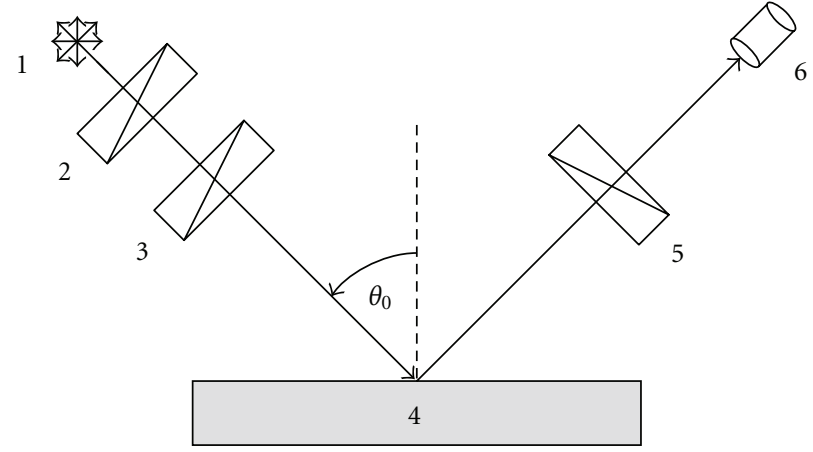

Figure 1: Simplified schematic diagram of the RPA ellipsometer, (1) unpolarized light, (2) fixed linear polarizer, (3) linear polarizer rotates at $\omega,(4)$ isotropic sample, (5) linear analyzer rotates at $\omega$, and (6) detector.

and the third harmonics. In this technique, it is feasible to extract the ellipsometric parameters $\psi$ and $\Delta$ from the even or odd terms without relying on the dc component which is considered to be a serious problem in RAE [10, 16, 17]. This allows measurements in a semidark room without worrying about stray light problems, dark currents in detectors, and long-term fluctuations in light sources. The proposed spectroscopic ellipsometer is applied to c-Si, and $\mathrm{Au}$.

\section{Theory}

A schematic diagram of the proposed ellipsometer is shown in Figure 1. It consists of the following elements: (1) light source (2) fixed linear polarizer with an azimuth angle $\theta$ (3) linear polarizer rotating at an angular speed $\omega(4)$ an isotropic sample (5) linear analyzer rotating at an angular speed $\omega$ and (6) photodetector. Here we assume that the azimuth angles of the rotating polarizer and the rotating analyzer are given by $P=\omega t+\tau$ and $A=\omega t+\delta$, respectively, where $\tau$ and $\delta$ are the azimuth angles of the rotating polarizer and the rotating analyzer at $t=0$, respectively. The azimuth angles $\theta, P$, and $A$ are referred to $\mathrm{p}$-axis of polarization. A well-collimated beam of monochromatic unpolarized light successively emerges through the fixed polarizer, the rotating polarizer, then is reflected from the sample, and finally is collected by the detector through the projection of the rotating analyzer as shown in Figure 1. The function of fixed polarizer is to remove the residual polarization of the light source and to increase the number of Fourier coefficients to four coefficients in order to avoid depending on the dc term.

Employing Jones-matrix formalism, the transmitted electric field $E_{t}$ in terms of the incident field $E_{i}$ is given by

$$
\begin{aligned}
{\left[\begin{array}{c}
E_{t} \\
0
\end{array}\right]=} & {\left[\begin{array}{cc}
\cos ^{2} A & \sin A \cos A \\
\sin A \cos A & \sin ^{2} A
\end{array}\right]\left[\begin{array}{cc}
r_{p} & 0 \\
0 & r_{s}
\end{array}\right] } \\
& \times\left[\begin{array}{cc}
\cos ^{2} P & \sin P \cos P \\
\sin P \cos P & \sin ^{2} P
\end{array}\right]\left[\begin{array}{c}
\cos \theta \\
\sin \theta
\end{array}\right] E_{i} .
\end{aligned}
$$

The intensity received by the detector is given by $I \propto\left|E_{t}\right|^{2}$. It is found that if the rotation angles of the polarizer and the 
analyzer are set to $P=\omega t+\tau$ and $A=\omega t+\delta$, respectively, then the intensity can be expanded as a Fourier series

$$
I(t)=g_{0}+\sum_{n=1}^{3} g_{n} \cos 2 n \omega t+\sum_{n=1}^{3} c_{n} \sin 2 n \omega t
$$

where $g_{0}, g_{n}$, and $c_{n}$ are Fourier coefficients.

Setting the azimuth angles $\theta, \tau$, and $\delta$ to zero reduces (5) to

$$
I(t)=g_{0}+g_{1} \cos 2 \omega t+g_{2} \cos 4 \omega t+g_{3} \cos 6 \omega t
$$

where the coefficients $g_{n}$ are related to the optical parameters by

$$
\begin{aligned}
& g_{0}=\frac{5}{4} \rho_{p}^{2}+\frac{1}{4} \rho_{p}^{2}+\frac{1}{2} \rho_{p} \rho_{s} \cos \Delta, \\
& g_{1}=\frac{15}{8} \rho_{p}^{2}-\frac{1}{8} \rho_{s}^{2}+\frac{1}{4} \rho_{p} \rho_{s} \cos \Delta, \\
& g_{2}=\frac{3}{4} \rho_{p}^{2}-\frac{1}{4} \rho_{p}^{2}-\frac{1}{2} \rho_{p} \rho_{s} \cos \Delta, \\
& g_{3}=\frac{1}{8} \rho_{p}^{2}+\frac{1}{8} \rho_{p}^{2}-\frac{1}{4} \rho_{p} \rho_{s} \cos \Delta .
\end{aligned}
$$

The ellipsometric parameters $\psi$ and $\Delta$ can be determined from three different sets of these coefficients. These sets are $\left(g_{0}, g_{1}, g_{2}\right),\left(g_{0}, g_{2}, g_{3}\right)$, and $\left(g_{1}, g_{2}, g_{3}\right)$. In our calculations, we will consider the third set because it doesnot contain the dc component. We will also compare the results obtained using this set with that of one of the first two sets. In terms of $g_{1}$, $g_{2}$, and $g_{3}, \tan \psi$ and $\cos \Delta$ can be written as

$$
\begin{aligned}
& \tan \psi=\frac{\sqrt{g_{1}+g_{3}}}{\sqrt{g_{1}-4 g_{2}+9 g_{3}}}, \\
& \cos \Delta=\frac{g_{1}-2 g_{2}-3 g_{3}}{\sqrt{\left(g_{1}+g_{3}\right)\left(g_{1}-4 g_{2}+9 g_{3}\right)}} .
\end{aligned}
$$

While using $g_{0}, g_{2}$, and $g_{3}, \tan \psi$ and $\cos \Delta$ can be written as

$$
\begin{aligned}
\tan \psi & =\frac{\sqrt{g_{0}+g_{2}}}{\sqrt{g_{0}-3 g_{2}+8 g_{3}}}, \\
\cos \Delta & =\frac{0.5 g_{0}-0.5 g_{2}-2 g_{3}}{\sqrt{\left(g_{0}+g_{2}\right)\left(g_{0}-3 g_{2}+8 g_{3}\right)}} .
\end{aligned}
$$

\section{Numerical Calculations and Error Analysis}

Simulated light signals are generated based on (4) without considering imperfections and misaligned optical components. Moreover, reflection and refraction at the planar interface between two isotropic media are assumed. The Fresnel complex reflection coefficients for $p$ and $s$ light components are calculated according to (3) based on the published values for the samples under consideration. In

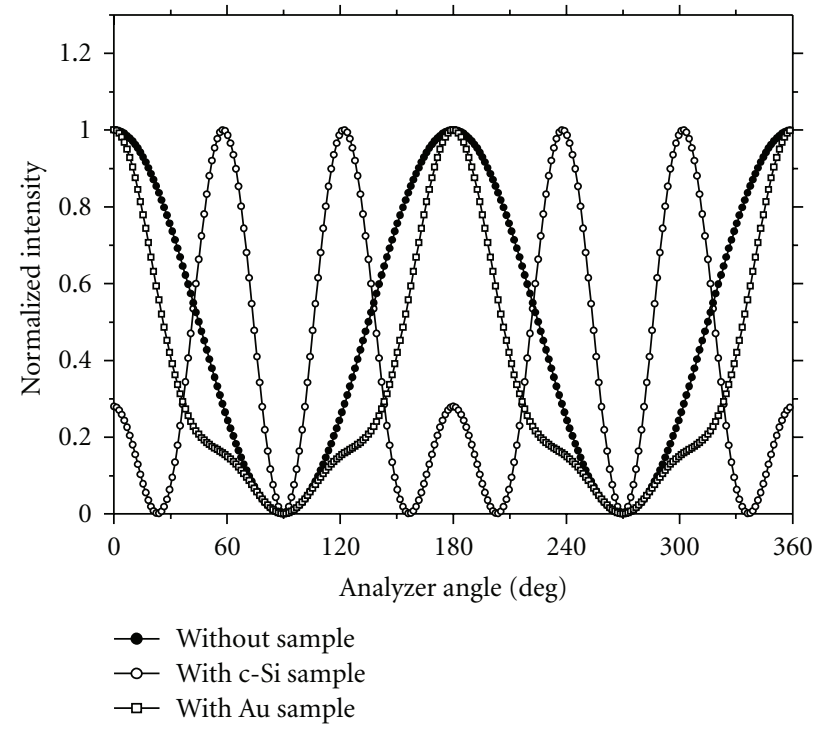

FIGURE 2: Normalized calculated light intensity as a function of the rotation angle of the analyzer in the presence of c-Si and Au samples and in the straight through situation with no sample at $\lambda=632.8 \mathrm{~nm}$ and $\theta_{0}=70^{\circ}$. The azimuth angles $\theta, \tau$, and $\delta$ are equal to zero.

the calculations below, we assume that the incidence angle $\theta_{0}=70^{\circ}$ and the azimuth angles $\theta, \tau$, and $\delta$ are set to zero.

Figure 2 shows the calculated light intensity, obtained from (4), using our model of RPA ellipsometer as a function of the rotation angle of the analyzer. The figure shows the calculated intensity when no sample is used and in the presence of c-Si and Au samples. Many interesting features can be seen in the figure. First, a substantial change in the intensity is observed in the presence of a sample. Second, the intensity goes to zero at the analyzer angles of $90^{\circ}$ and $270^{\circ}$. This is attributed to the crossing of the fixed and the first rotating polarizer. This condition along with (6) imposes that the coefficients are related to each other by the relation: $g_{0}-g_{1}+g_{2}-g_{3}=0$. Third, the intensity shows maxima at specific angles of $\left(0^{\circ}, 180^{\circ}, 360^{\circ}\right)$ in all cases due to the transmission axes of the fixed and the first rotating polarizer being parallel. Moreover, new features arise in the presence of a sample. These features depend on the wavelength, the angle of incidence, and the optical parameters of the sample.

3.1. Noise Effect. The ultimate accuracy and detection limit of an ellipsometer is determined by the inevitable presence of undesired noise which causes random fluctuations in the recorded signal. This noise may originate from a variety of sources such as thermal fluctuations in the light source, mechanical vibrations, pickup of stray AC light, and the self-generated noise in detectors and electronics. Such noise is reduced by signal averaging and can be measured by performing multiple identical runs and by calculating the mean and the standard deviations.

In order to simulate real signals, noise was generated using Mathcad code and was superimposed on a clean signal 


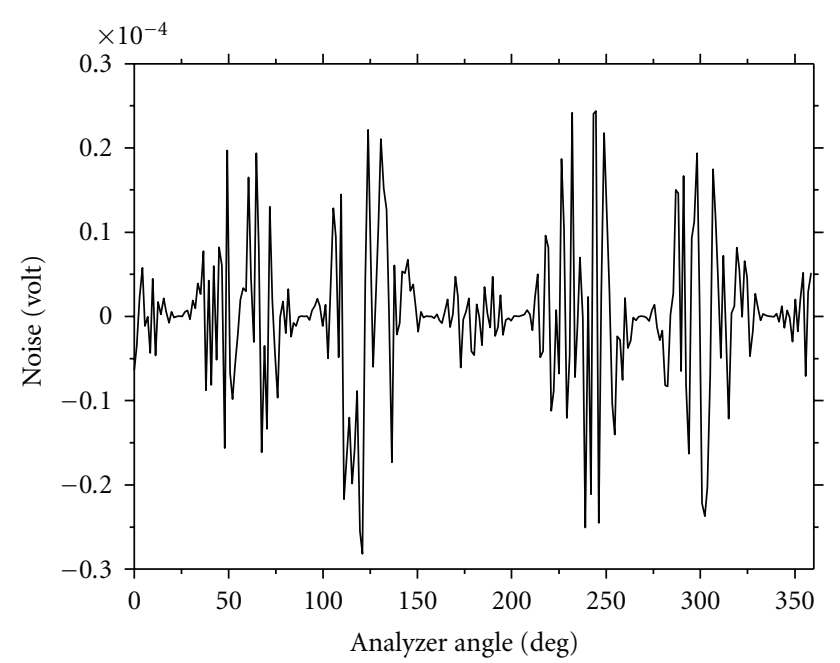

FIGURE 3: The noise superimposed on the clean signal.

according to the following equation:

$$
I_{\text {noise }}=\left(\operatorname{rnd}(c)-\frac{c}{2}\right) I+\left(\operatorname{rnd}(e)-\frac{e}{2}\right)+0.0001 I_{\max },
$$

where Mathcad's $\operatorname{rnd}(c)$ function produces random noise in the range from 0 to $c$ and $\operatorname{rnd}(e)$ function produces random noise in the range from 0 to $e$. In our simulation, we consider $c=e=1$. The first term represents the random noise recorded by the detector due to the thermal fluctuations of the light source after passing through the system, the second corresponds to Johnson noise and shot noise encountered in the detector and readout electronics, and the third term represents the dc offset due to long time drifts. Figure 3 shows the noise superimposed on the clean signal shown in Figure 2 at a wavelength $632.8 \mathrm{~nm}$ for c-Si.

The calculations of the dielectric function of $\mathrm{c}-\mathrm{Si}$, are based on the well-known equation [1]

$$
\varepsilon=\sin ^{2} \theta_{0}+\sin ^{2} \theta_{0} \tan ^{2} \theta_{0}\left(\frac{1-\rho}{1+\rho}\right)^{2},
$$

where $\rho=r_{p} / r_{s}=\tan \psi e^{i \Delta}, \varepsilon=\varepsilon_{1}+i \varepsilon_{2}, \tilde{n}=\sqrt{\varepsilon}=n+i k$, $\varepsilon_{1}=n^{2}-k^{2}$, and $\varepsilon_{2}=2 n k$.

In our calculations, the published data are used to simulate the output signal for a given sample and then this signal is used to reconstruct back the optical constants. The published and the calculated real and imaginary parts of the refractive index of c-Si and Au using the sets $\left(g_{0}, g_{2}, g_{3}\right)$, and $\left(g_{1}, g_{2}, g_{3}\right)$ are depicted in Figure 4 . The data used in these calculations were that of Palik [18]. As can be seen from the figure, the reconstructed data based on the proposed model for the set $\left(g_{1}, g_{2}, g_{3}\right)$ are in good agreement with the original data. The calculations using the set $\left(g_{0}, g_{2}, g_{3}\right)$ reveal a considerable error particularly in the values of $k$ for $\mathrm{c}-\mathrm{Si}$.

The insets shown in Figure 4 are plotted using the set $\left(g_{1}, g_{2}, g_{3}\right)$. They show that at energies below $2.5 \mathrm{eV}$ there are

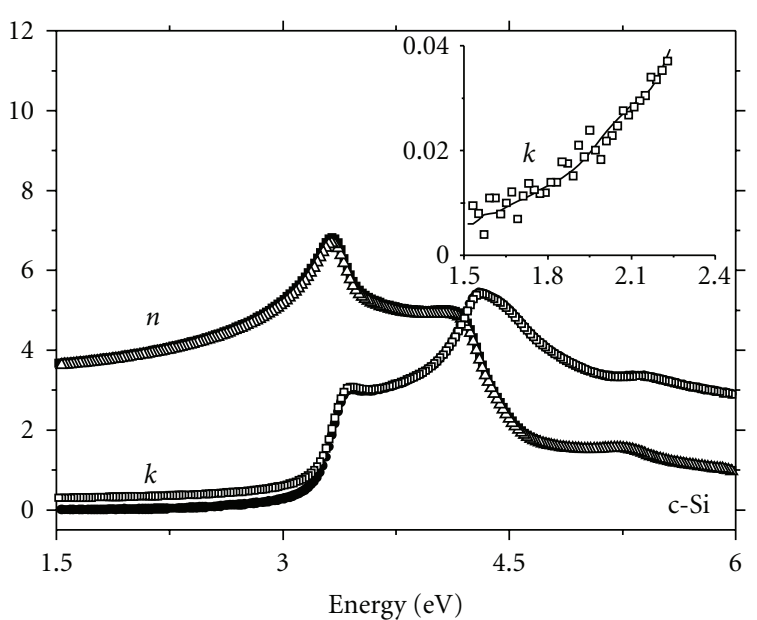

(a)

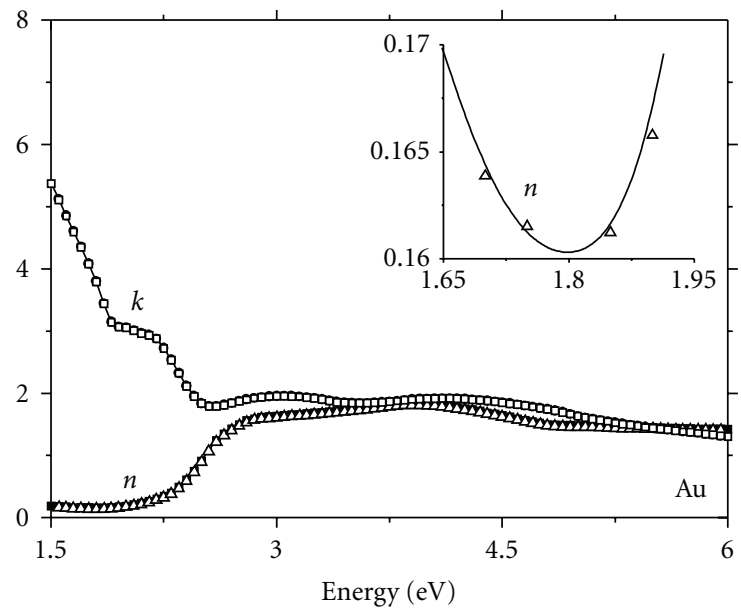

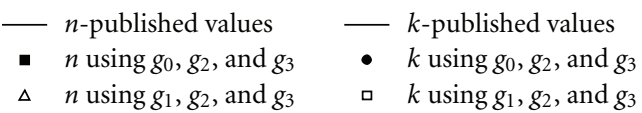

(b)

Figure 4: Complex refractive index of $\mathrm{c}-\mathrm{Si}$ and $\mathrm{Au}$, in the range from 1.5 to $6 \mathrm{eV}$. Lines: published values, Points: calculated values with noisy signal using two sets of the coefficients.

coniderable fluctuations in the values of $k$ and $n$ for c-Si and $\mathrm{Au}$, respectively, due to the noise imposed on the clean signal. Such behavior is attributed to the very small values of $k$ for c-Si and $n$ for $\mathrm{Au}$ in this region. Most of the ellipsometers which donot include phase retarders suffer such a problem [19].

The percent error in the calculated values of $n$ and $k$ for $\mathrm{c}-\mathrm{Si}$ and $\mathrm{Au}$ is shown in Figure 5 using the set containing $\left(g_{1}, g_{2}, g_{3}\right)$ and that containing $\left(g_{0}, g_{2}, g_{3}\right)$. These fluctuations shown in the figure are due to the noise imposed on the clean signal as mentioned before. It is clear that the set containing the dc term has a considerable impact on the values of the optical parameters of the two samples while the other set produces much lower percent error. It is worth to mention 


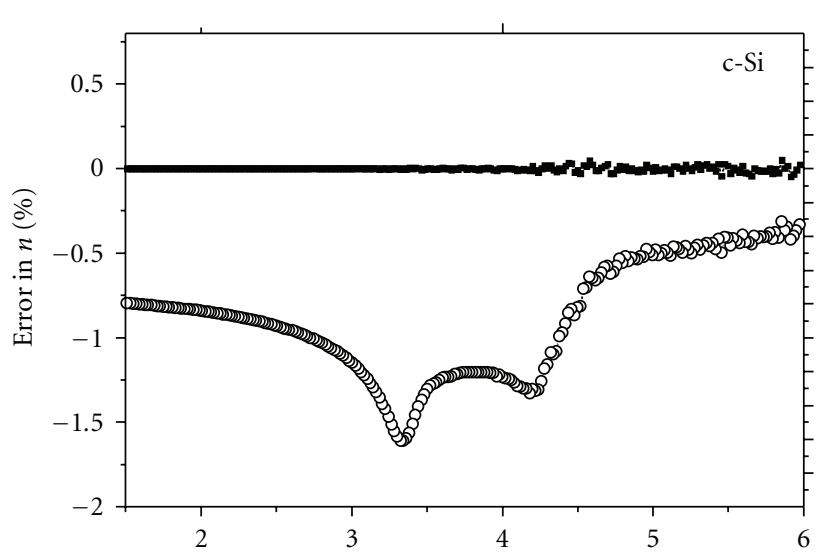

(a)

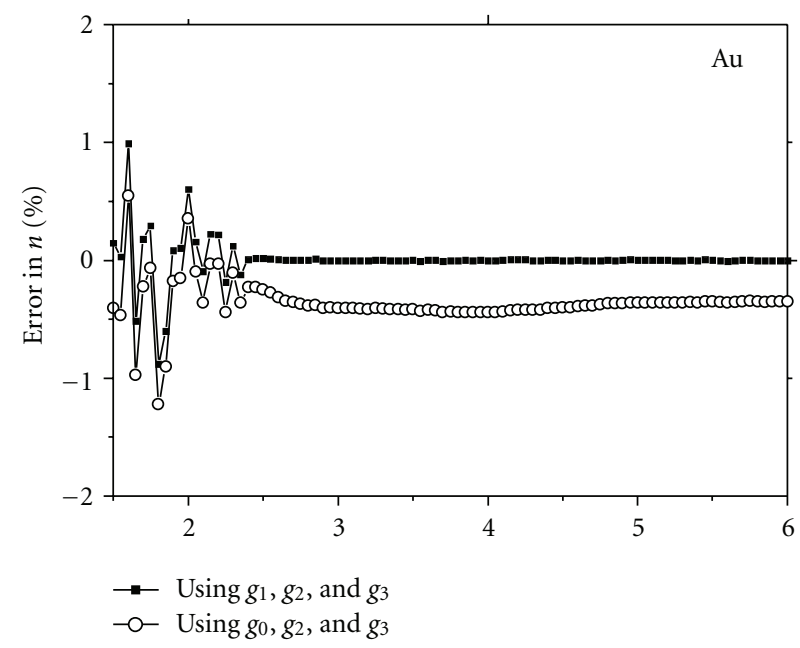

(c)

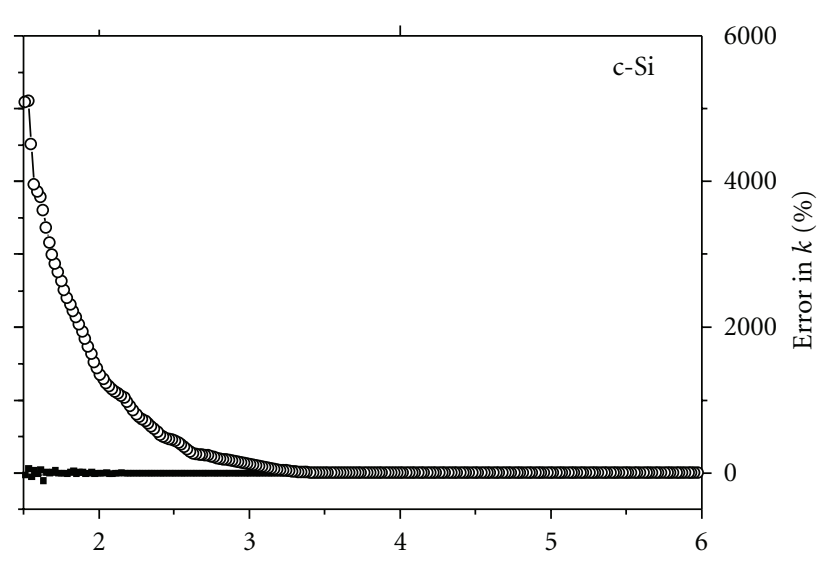

(b)

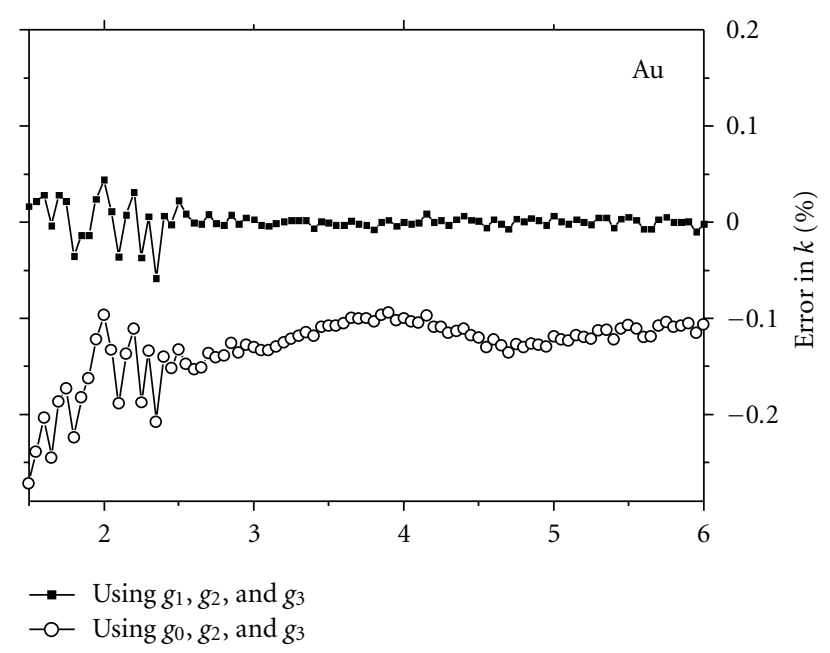

(d)

FIGURE 5: Percent error in the calculated values of $n$ and $k$ for Au and c-Si using the proposed RPA ellipsometer using the set containing $\left(g_{1}, g_{2}, g_{3}\right)$ and that containing $\left(g_{0}, g_{2}, g_{3}\right)$.

that the dc offset of the signal is considered to be a major problem in ellipsometers that rely on this term. On the other hand, the proposed ellipsometer has the advantage that the results do not depend entirely on this term.

3.2. Systematic Errors. Some sources of systematic errors are due to the azimuthal misalignment of optical elements with respect to the plane of incidence, sample mispositioning, beam deviation, and collimation errors. Wavelength and angle of incidence errors, also, contribute to systematic errors. Thus, it is necessary to invoke such error analysis in our model. These errors can be significantly reduced by careful alignment and calibration of the system and by choosing high quality optical components.

Thus, it is important for the verification process to have some quantities for determination of the accuracy of the simulated data as a result of misalignment of the fixed polarizer, rotating polarizer, and rotating analyzer. The parameters to be checked are the ellipsometric parameters $\psi$ and $\Delta$ as well as the real and the imaginary parts of the index of refraction $n$ and $k$.

Figure 6 shows the percent error committed on $\psi, \Delta$, $n$, and $k$ of $\mathrm{c}-\mathrm{Si}$ sample as a function of the errors of the fixed polarizer azimuth angle $\theta$, the rotating polarizer azimuth angle $\tau$, and the rotating analyzer azimuth angle $\delta$ successively varied from $-0.2^{\circ}$ to $0.2^{\circ}$ in steps of $0.02^{\circ}$ while keeping the two other variables equal to zero. As can be seen from the figure, the impact of these errors on $\psi, \Delta$, and $n$ is not significant for small misalignment. On the other hand, it is considerable for $k$ since it has a small value $(\sim 0.019)$ at a wavelength of $632.8 \mathrm{~nm}$ [18]. The figure also reveals that the error in azimuth angle $\delta$ has almost the lowest impact on the four parameters under consideration.

The sensitivities of $\tan \psi$ and $\cos \Delta$ to the coefficients $g_{1}, g_{2}$, and $g_{3}$ are shown in Figure 7 for c-Si in the photon range of $1.5-6 \mathrm{eV}$. The figure shows the well-known results; 


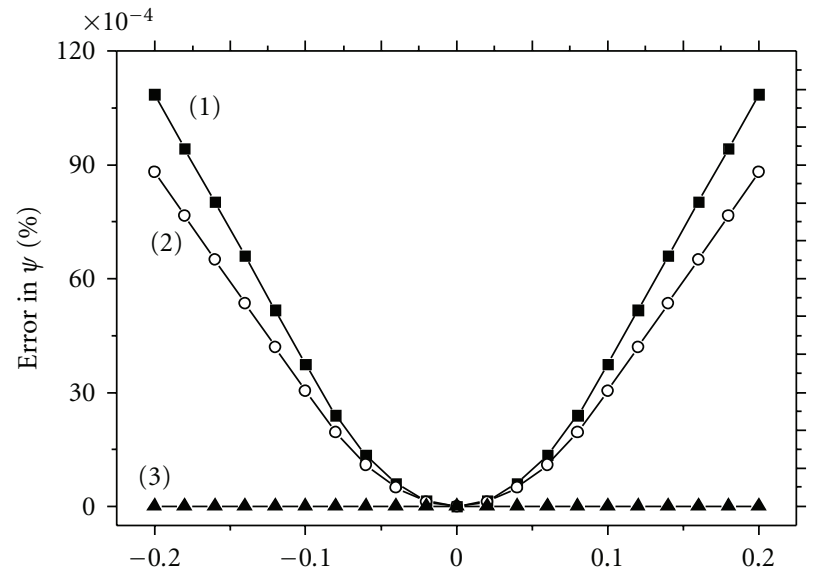

(a)

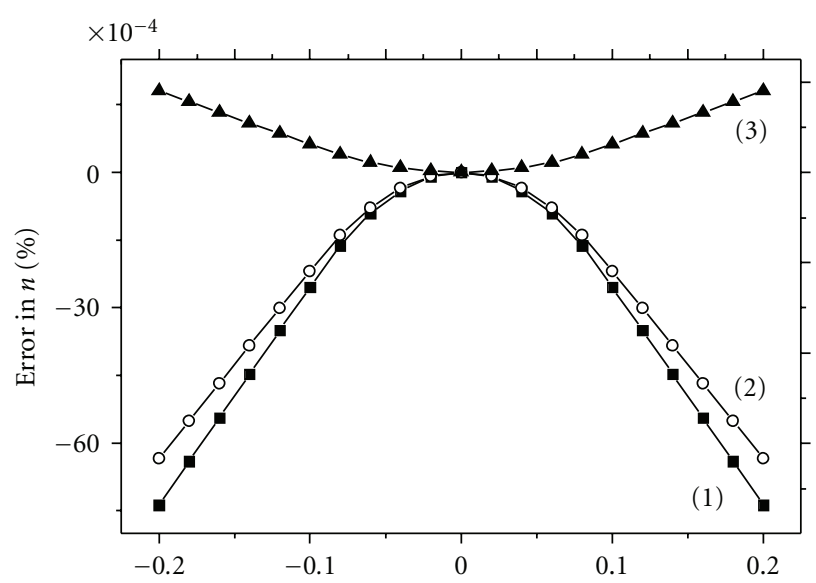

(c)

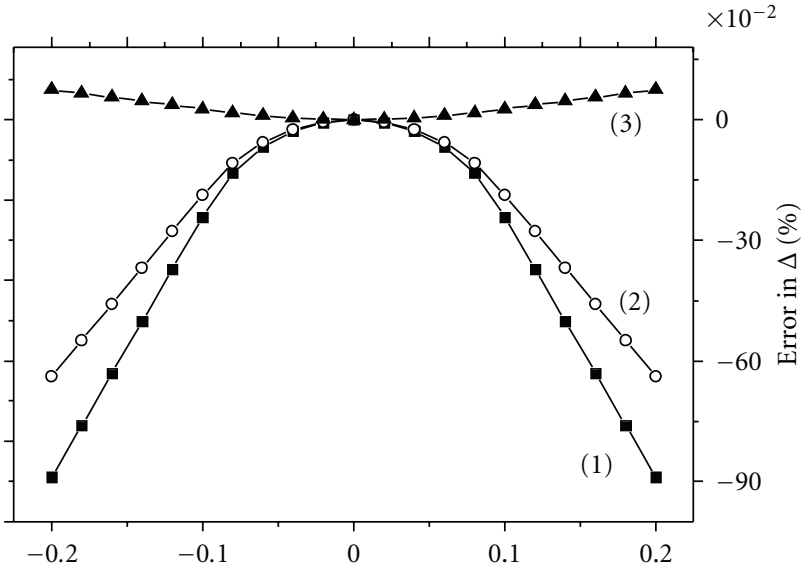

(b)

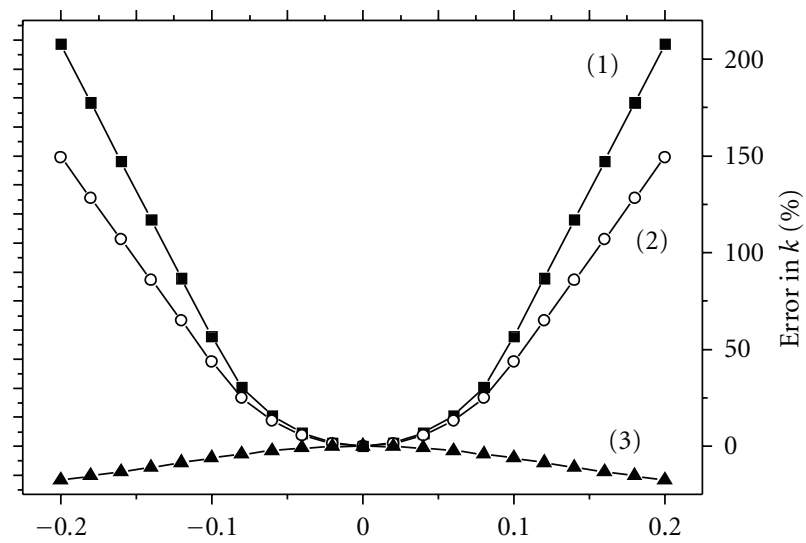

(d)

Figure 6: Percent error in $\psi, \Delta, n$, and $k$, for Si sample at $\lambda=632.8 \mathrm{~nm}$ and $\theta_{0}=70^{\circ}$, as a function of the error in (1) $\theta$, (2) $\tau$, and (3) $\delta$ successively varied from $-0.2^{\circ}$ to $0.2^{\circ}$ in steps of $0.02^{\circ}$ while keeping the two other variables equal to zero.

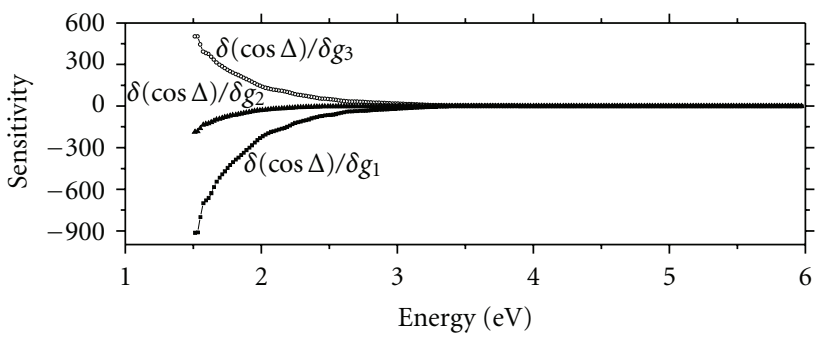

(a)

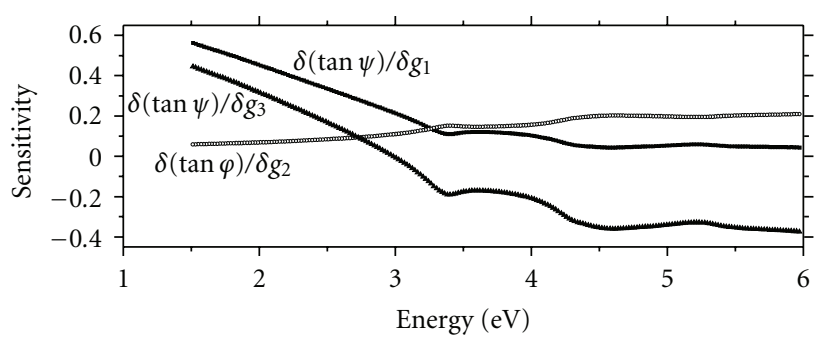

(b)

FIGURE 7: Dependence of the derivatives of (a) $\cos \Delta$ and (b) $\tan \psi$ on energy for c-Si.

at low energies, where c-Si is essentially transparent and the sensitivity is high compared to the high-energy region. A compensator is suggested to solve such a problem [19]. As can be seen from the figure, $\Delta$ is very sensitive to $g_{1}$ and $g_{3}$ with an important feature: the sensitivity to $g_{3}$ is positive whereas it is negative to $g_{1}$. This effect has the advantage of reducing the whole sensitivity of $\Delta$.

\section{Conclusion}

A scanning ellipsometer by rotating the polarizer and the analyzer in the same direction at a speed ratio of $1: 1$ has been proposed. The validity of this ellipsometer has been demonstrated theoretically for two samples: $\mathrm{c}-\mathrm{Si}$ and Au. The calculated optical parameters for the two samples are found 
to be in good agreement with original published data in the photon energy $1.5-6 \mathrm{eV}$. The results of our model do not depend on the dc offset of the signal which is considered the most important advantage of the proposed RPA.

\section{References}

[1] R. M. Azzam and N. M. Bashara, Ellipsometry and Polarized Light, North-Holland, Amsterdam, The Netherlands, 1977.

[2] P. S. Hauge, "Recent developments in instrumentation in ellipsometry," Surface Science, vol. 96, no. 1-3, pp. 108-140, 1980.

[3] J. A. Woollam, P. G. Snyder, and M. C. Rost, "Variable angle spectroscopic ellipsometry: a non-destructive characterization technique for ultrathin and multilayer materials," Thin Solid Films, vol. 166, pp. 317-323, 1988.

[4] K. Vedam, "Spectroscopic ellipsometry: a historical overview," Thin Solid Films, vol. 313-314, pp. 1-9, 1998.

[5] D. E. Aspnes, "Expanding horizons: new developments in ellipsometry and polarimetry," Thin Solid Films, vol. 455-456, pp. 3-13, 2004.

[6] D. E. Aspnes, "Fourier transform detection system for rotating-analyzer ellipsometers," Optics Communications, vol. 8, no. 3, pp. 222-225, 1973.

[7] D. E. Aspnes and A. A. Studna, "High precision scanning ellipsometer," Applied Optics, vol. 14, no. 1, pp. 220-228, 1975.

[8] A. R. M. Zaghloul and R. M. A. Azzam, "Single-element rotating-polarizer ellipsometer: PSI meter," Surface Science, vol. 96, no. 1-3, pp. 168-173, 1980.

[9] L. Viña, C. Umbach, M. Cardona, and L. Vodopyanov, "Ellipsometric studies of electronic interband transitions in $\mathrm{Cd}_{x} \mathrm{Hg}_{1-x}$ Te," Physical Review B, vol. 29, no. 12, pp. 67526760, 1984.

[10] L. Y. Chen and D. W. Lynch, "Scanning ellipsometer by rotating polarizer and analyzer," Applied Optics, vol. 26, no. 24, pp. 5221-5228, 1987.

[11] L. Y. Chen, X. W. Feng, YI. Su, H. Z. Ma, and Y. H. Qian, "Improved rotating analyzer-polarizer type of scanning ellipsometer," Thin Solid Films, vol. 234, no. 1, pp. 385-389, 1993.

[12] L. Y. Chen, X. W. Feng, YI. Su, H. Z. Ma, and Y. H. Qian, "Design of a scanning ellipsometer by synchronous rotation of the polarizer and analyzer," Applied Optics, vol. 33, no. 7, pp. 1299-1395, 1994.

[13] C. Wijers, "A one-wavelength, in situ alignment method for rotating analyser ellipsometers," Applied Physics B Photophysics and Laser Chemistry, vol. 27, no. 1, pp. 5-8, 1982.

[14] R. M. A. Azzam, "A simple Fourier photopolarimeter with rotating polarizer and analyzer for measuring Jones and Mueller matrices," Optics Communications, vol. 25, no. 2, pp. 137-140, 1978.

[15] I. An, M. G. Park, K. Y. Bang, H. K. Oh, and H. Kim, "Development of multichannel ellipsometry with synchronously rotating polarizer and analyzer," Japanese Journal of Applied Physics, Part 1, vol. 41, no. 6 A, pp. 3978-3980, 2002.

[16] R. Greef, "An automatic ellipsometer for use in electrochemical investigations," Review of Scientific Instruments, vol. 41, no. 4, pp. 532-538, 1970.

[17] W. Budde, "Photoelectric analysis of polarized light," Applied Optics, vol. 1, pp. 201-205, 1962.

[18] E. D. Palik, Handbook of Optical Constants of Solids, Academic Press, San Diego, Calif, USA, 1985.
[19] J. Opsal, J. Fanton, J. Chen et al., "Broadband spectral operation of a rotating-compensator ellipsometer," Thin Solid Films, vol. 313-314, pp. 58-61, 1998. 

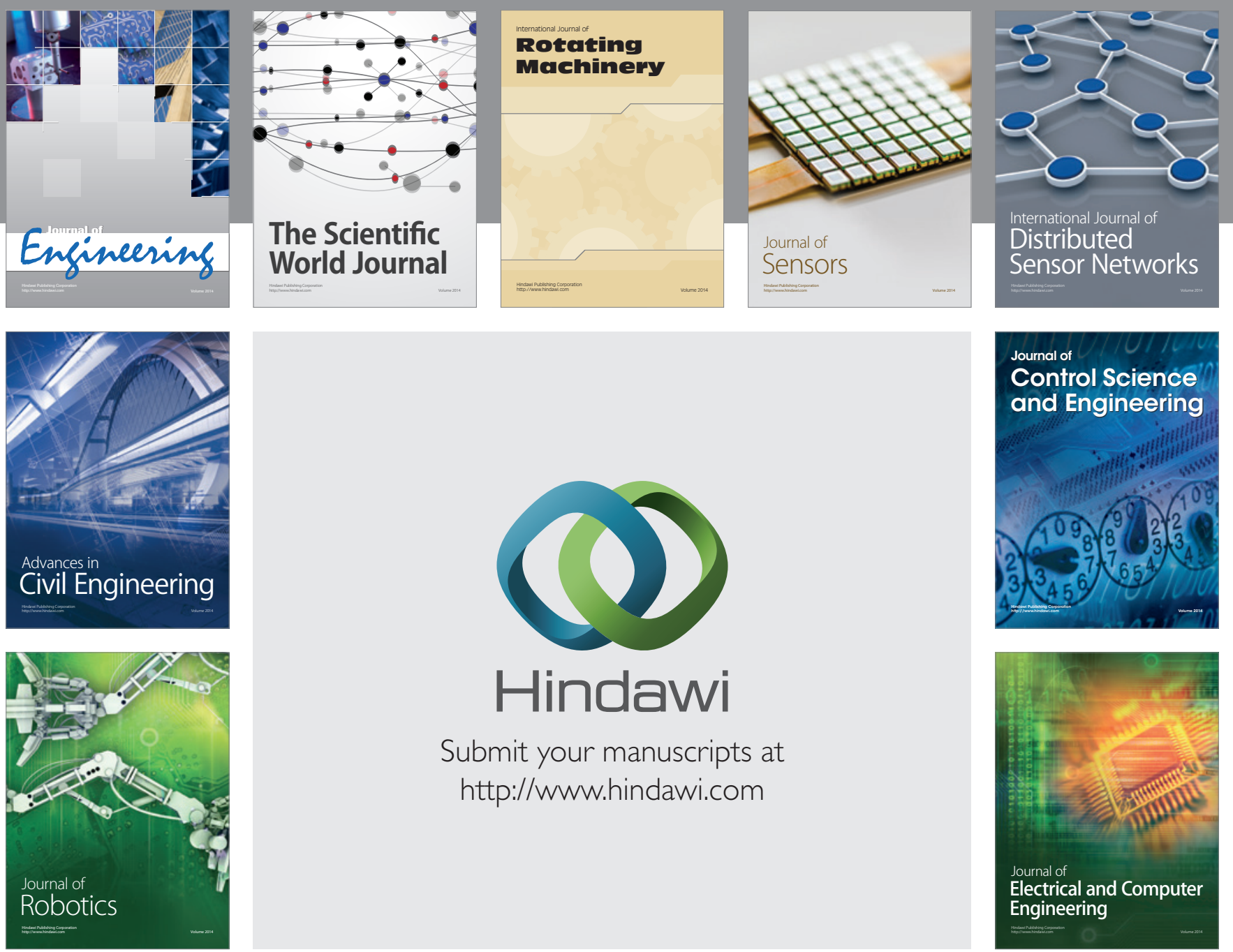

Submit your manuscripts at

http://www.hindawi.com
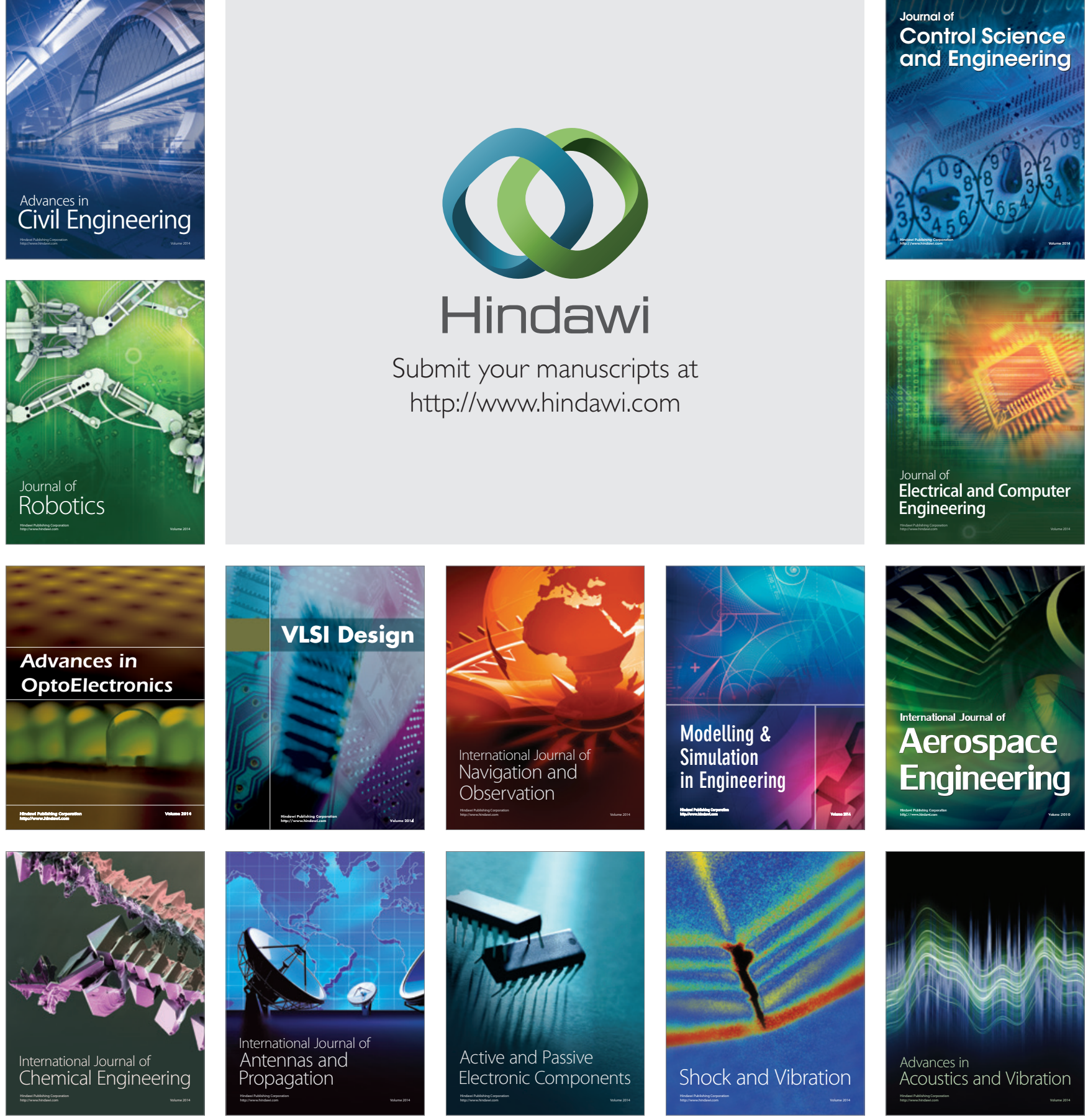